\title{
REVIEW OF MODELING AND STIMULATING HUMAN IMMUNE SYSYTEM
}

\author{
Hongwei Mo \\ Automation College, Harbin Engineering University Harbin, 150001, China
}

Abstract: The human immune system(HIS) is a self-adaptive, complex system. Many different components cooperate each other to emerge intelligence behavior. Many researches have been done in order to explore the nature of immune system and its intelligent behavior. Computational models and concept models are the main two approaches of modeling and simulating the HIS. They are reviewed in detail in this paper. Differential equation and cellular automaton are two ways of being used frequently in modeling and simulating the HIS. The advantages and disadvantages of them are discussed in order to inspire new ways of researching the intelligent behavior of it. The application of HIS modeling in $\mathrm{AI}$ is also introduced briefly .

Key words: Human Immune System, Modeling, Simulating

\section{INTRODUCTION}

The HIS is a complex set of cells and molecules distributed throughout the body. It provides a natural deference mechanism from the infection agents such as viruses, bacteria, fungi or other parasites. As explained in Jerne [14] the HIS has the ability to memorize the foreign substances that enter the body. The whole set-up represents a very complex and dynamic system that is distributed over billiards of elements but still maintaining effective communication.

The basic component of the HIS is lymphocyte (white blood cells). There are two types: B-lymphocyte (produced in bone marrow) and T-lymphocyte (trained in thymus). Then there are other elements such as antigen presenting cells (APC) identifying antigens, natural killer cells (NK cells), antibody 
molecules and message molecules (lymphokines). In the past decades, research in Artificial Intelligence has been inspired by phenomena observed in life. The HIS shares many properties with neural networks and genetic algorithms. Modeling and Simulating the HIS in a computer provides immunologists with a new way of testing immunological theories, experiments in labs. However, if we observe the HIS and models of the HIS from an artificial intelligence or a complex systems simulation point of view, a number of questions arise.

Are techniques from the HIS simulation applicable to artificial intelligence or scientific computing? What properties of the HIS make it particularly fit or unfit to use the HIS as a natural solver?

In the past years, artificial intelligence researchers have started using models of HIS as a base for developing new artificial intelligence techniques. In the paper, a review of approaches of modeling and simulating HIS in recent years is given in order to inspire new ways of designing intelligent system.

The paper is organized as follows. In section 2, many approaches of modeling and simulating the HIS are reviewed and analyzed. The applications of HIS models are given in section 3. Discussions and conclusions are given in section 4 and 5 respectively.

\section{APPROACHES OF MODELING AND SIMULATING THE HIS}

Some models are referred to identify the computational model, i.e. the algorithm or the code, while the other models to the conceptual model in term of logical statements describing the entities and their interaction rules in the following sections.

\subsection{Computational His Models}

\subsubsection{Differential Equation}

The earliest models of the HIS are differential equation based models. They have been very popular, and a wide range of immunological phenomena have successfully been simulated using differential equation based models. A list of titles and abstracts of publications using a differential equations based model of HIS is composed in[15].

Most of these models focus on one specific phenomenon observed in immunology. The outcome of the model is then compared to data of in vivo 
or in vito experiments. Differential equation models of the HIS have been very successful for immunologists. From the viewpoint of AI, the following properties make differential equation based models less appropriate.

- Only theories involving a small number of variables can be modeled

- The diversity of the HIS can not easily be modeled

- Incorporating theories about different phenomena in HIS in the same model is hard, because the relations between those parts are not easily expressed in differential equations

The operation of differential equation based models is very different from what happens in reality. While differential equation based models are used widely in all research areas that simulate complex systems, they are of little interest to artificial intelligence, since they depend on known relations between variables, while artificial intelligence focuses on areas where these relations are unknown.

\subsubsection{BIT-String Based Models}

In a real HIS, binding between for example a B-cell receptor and an antigen's B-cell epitope will take place through the matching of the amino acids string. It is possible to represent the amino acid string using an alphabet of $m$ characters where each character corresponds to a given amino acid. Then, any of a number of string-matching algorithms can be used to determine the degree of complementarity. For a string of length $\mathrm{N}$, the repertoire size is given by $m^{N}$ such receptor's or epitope's idiotype was first introduced by Farmer et al[9] as a way to perform clever calculations for determining molecular complementarity and predict the optimal size of an epitope. But the greatest impact the string representation introduced by Farmer is in lattice gas simulations of the HIS. The typical lattice gas simulations chose to build the epitope and receptor strings using an alphabet containing only two characters, namely 0 and 1 , and those models are consequently referred to as 'bit-string' models. Since the receptors and epitopes of a bit-string model are represented by a string of bits,i.e. a string of $0 \mathrm{~s}$ and $1 \mathrm{~s}$, those models have a repertoire size of $2^{N}$ is the length of the bit string.

\subsubsection{Genetic Algorithm}

Another very interesting research is coming up with interesting results. The research is based on the work of the University of New Mexico and is mainly composed of Forrest et al. They have published very interesting papers using the bit-string representation where they explore evolution and somatic learning in V-region genes[21], and the use of genetic algorithms to 
explore pattern recognition in the HIS[10].In[21], they found that their 512bits genome can encode information about 32 64-bits antigens, i.e.about 2048 bits. The organization of the resources by the HIS suggests that the system has the ability to recognize pattern in the antigens and exploits the HIS to maximize its recognition while minimizing its resources. In[10], they explored the HIS further using genetic algorithm and found that it was impressively good at recognizing patterns. Challenging the pattern recognition capabilities of the HIS, they found that the genetic algorithm together with clonal selection could evolve an antibody type that matched multiple antigens through the identification of common schemes among antigens. They also found that when the genetic algorithm was faced with 2 or 3 different antigens, i.e., multiple peaks in the antigen landscape, the genetic algorithm can discover all peaks and similarity among antigens does not prevent distinction by antibodies, no matter how similar the antigens are. Finally, they found that the number of antibodies exposed to any antigen will greatly affect the capacity of the HIS to recognize patterns. But perhaps even more interesting is the group's attempt at constructing a computer immune system to improve computer security[25].

\subsubsection{Large Scale Simulation}

Cellular Automata based models have proven capable of providing several new insights into the dynamics of the HIS response. A qualitative picture of the HIS behaviors can be obtained with small-scale simulations. However for a more detailed analysis and to further validate the models, large scale simulations are required.

One of the most prominent attempts to cope with the quest for biological fidelity is the IMMSIM (Immune Simulator or CS-model) automaton, developed by Seiden and. Celada in 1992[23]. It is also the first cellular automaton model using bit-strings to model the receptors and epitopes.

IMMSIM is a very interesting model as it is attempting to create a general HIS simulator rather than only a simplified toy model for answering a particular problem. The various authors who have used the model claim that the results obtained compared well with experimental data. The model has already been used to investigated various immunological questions[6][3][7][1]. Those results were obtained by the IMMSIM group based at Princeton University and their collaborators. This new approach is closer to the classical definition of cellular automata than the previous one. It has the advantage of achieving the same results obtained with the IMMSIM with a lower computational effort. IMMSIM belongs to the class of immunological cellular automata, but its degree of sophistication sets it apart from simpler CA in the Ising-like class[16][19]. 
Bernaschi and Castiglione came up with a parallel coded version of the latest IMMSIM entirely written in the $C$ language that they called ParImm[2] and later CIMMSIM. CIMMSIM was designed according to criteria of openness and modularity to allow smooth upgrades and addition of new features (cells, molecules, interactions and so on) for future investigations. The current version of the code (version 4.3) is able to simulate both the humoral response described in[23][4] and the cellular response described in [5]. In the respect it is the most advanced parallel version of the IMMSIM.

In[18], Meier-Schellershiem and Mack present their own model, SIMMUNE. The model is run on a 3-D grid and can be made to comprise different compartments. It lets the user define the properties of the compartments with the simulation such as the dimension of the compartment, the diffusion rates of molecules and cells with the compartments and the initial concentrations of the different types of agents can be given. Further, the exchange of agents between the different compartments can be regulated. Interestingly, the model incorporates the ability of B-cells to respond to certain kind of antigens directly, i.e. without the assistance of $T$ helper cells. One major drawback of the SIMMUNE model is that it is impossible to find any kind of precise description of its implementation.

\subsection{Concept Models}

\subsubsection{Agent-Based Models}

The goal of [22] is to present an agent-based, bottom-up approach to the HIS modeling. This approach is widely used in Artificial Life. The HIS is a suitable candidate for this kind of modeling because it has a structure of noncentralized system based on a high number of distributed elements. The set of local level activities results in a well working. It presents an example of bottom-up HIS model and discusses its advantages and disadvantages. The model is focused on visualizing the dynamic interactions in the system. A bottom-up approach is based on the synthesis of a complex system from the activities on a lower system level. The artificial models have a design of a computer environment with a set of simple autonomous entities on local a level. The activity of these entities is emerging into the behavior of a global system. A model with this kind of structure is easily implemented by using agents-based modeling. It offers us a direct "entity to agent" implementation of entities' features. An agent-based modeling, in general, offers a tool for biological systems modeling that is easy to understand and develop. 


\subsubsection{Swarm-Based Models}

In[13], it presents a swarm-based, 3-dimensional model of the HIS and its response to first and second viral antigen exposure. The model utilizes a decentralized swarm approach with multiple agents acting independentlyfollowing local interaction rules - to exhibit complex emergent behaviour, which constitute externally observable and measurable immune reactions. The two main functional branches of the human immune system, humoral and cell-mediated immunity, are simulated in the model. It models the production of antibodies in response to a viral population. Antibody-antigen complexes are formed, which are removed by macrophages. This system also demonstrates reinforced reaction to a previously encountered pathogen, thus exhibiting realistic memory response.

\subsubsection{Cellular Automaton}

Many models of the HIS using a cellular automaton are based on the work of de Boer [8]. In 1992, de Boer et al. used a cellular automaton to model HIS. Their model is based on Jerne's network theory, which is a classic immunology theory described by differential equation, and simulated the activation of B-cells caused by injection of antigen.

According to Jerne's network theory, the main cause of immunity is the emergence of a network of interacting B-cell populations.

The cellular automata models [8][24][26][27] based on the theory, model the so called shape space of the B-cell receptors. In these models, B-cells with every possible receptor are represented as cells in a multidimensional grid. The shape of a receptor of a B-cell is represented by the vector denoting the grid position of the B-cell. Two B-cells have a matching receptor if their position vectors are opposite. A cell can be in two states, active or non-active.

In a cellular automaton, the state of a cell at time $t+1$ depends only on the state of its most direct neighbours at time $t$, or earlier. Usually, if the position of a cell in the grid is denoted by a vector $a$, its neighbours are those cells with a position close to $a$. The cellular automaton models of HIS use a different concept of neighbourhood, that expresses the concept of affinity between two receptors. Many variants of the basic model have been constructed, some of which use more than two states, others using different numbers of dimensions. A serious problem with these cellular automaton based models is that they are still based on the immune network theory.

It may be worth mentioning the work in[29], which have developed simplistic CA toy models of HIS to answer specific questions. In [29] it uses a very simple celluar automaton of immunization and aging in the context of immune network. The cellular automaton model is such that rather than 
having different types of cells moving around on the grid(lattice gas model), every site of the CA grid in a d-dimensional space corresponds to the shape of a molecule(associated with a B cell or antibody) and can be in any of 3 states: $B_{i}=0$ (or nonactivated) for low, $B_{i}=1$ for immediate or $B_{i}=2$ for high(activated). The authors then define a field, $h_{i}$ that represents the concentrations of complementary receptors as $h_{i}(t)=\sum_{2 d+1} B_{i}(t)$. The rule
for the evolution of the system is then defined as

$$
B_{i}(t+1)=\left\{\begin{array}{l}
B_{i}(t)+1 \text { if } \theta_{1} \leq h_{i}(t) \leq \theta_{2} \\
B_{i}(t)-1 \text { otherwise }
\end{array}\right.
$$

But no change is made if it would lead to $B_{i}=-1$ or 3 . This simplistic model has a transition region, between a stable and chaotic region, where the authors have observed emergent complex behavirour which they claim is appropriate to describe a self-regulated multiconnected network and possibly provides the much needed connections between the available experimental results and the Jerne's theory.

HIS models using a modified cellular automaton have several advantages[12]:

Firstly, it can estimate the distribution of behaviors more widely, not only the average because of stochastic characteristics.

Secondly, it is easy to modify the complexity of the interactions without introducing any new difficulties in solving the model because nonlinear is not intrinsically difficult to treat in cellular automata.

Lastly, the cellular automaton is able to represent the components and processes of interest in biological language. Thus can allow researchers to check the correctness of the model.

However, there are penalties to be paid. First we will always be dealing with a relatively small system. In reality, the HIS is gigantic, in humans involving $10^{12}$ cells of perhaps $10^{9}$ possible specificities. The simulations will be always much smaller and researchers must pay constant attention that the results they see are not artifacts due to our restricted size. Second it will sometimes be hard to extract the essence of what is happening.

\section{APPLICATIONS OF THE HIS MODELS}

One hand,currently many applications can be found by using ideas derived from the HIS and its simulation in the field of medical science.

On the other hand, many properties of the immune system have been recognised as important features for a number of applications in the field of computer science or Aritifial Immune Systems(AIS). In the last few years, a number of applications of artificial immune systems had been developed. 
Many applications have been in the field of computer security[25], robot control [28] or semiconductor production line[11]. Some applications use models based on Jerne's network theory. Other applications use general properties of the immune system that are considered useful for the problem that is being researched, and implement them in ways very different from the HIS.

If we observe the immune system from an artificial intelligence point of view, we can see it as a learning system that autonomously performs a number of tasks including pattern recognition, learning from examples, regulating actions based on sensor information, and optimization toward better solutions[12]. Thus, the immune system consists of several learning components. For artificial intelligence research, interesting properties of the immune system are the following.

- Classification: discrimination between self and non-self

- Regulation: Control of the reaction against antigen

- Parallelism: Distributed memory for known antigen

- Optimization: Adaptive recognition leading to a faster response

The Celada and Seiden model exhibits all of these properties. Since the model classifies data that is represented by bit strings, it could be used in various classification and pattern recognition tasks. Representing the problem data as bit strings in a way that is usable and meaningful in the CS model.

\section{DISCUSSION}

Computational modeling is of growing interest in a number of fields including biology, mathematics, physics and computer science.

We have to distinguish between two types of computer modeling, namely computational models[12][17], such as differential equation and concept models, such as cellular automaton. As mentioned above, the former is often a preferable way to model a complex system since more is know about differential equations. It is often the case that the differential equations will likely contain nonlinear and time-delays. The simplifications made are of mathematical concern and will most often distort the dynamics away from that of the real system. Of course, if one is to model the HIS using differential equations, common sense dictates that one has to first simplify the dynamics to no more than a few equations. It is not always a bad approach as it will, for a valid model, give an averaged estimate of the dynamics.

But when one's ultimate goal is to understand the full behavior of the system, in particular the characteristics of the local rules that give rise to the 
formidable array of complex behavior observed in HIS, it is necessary to resort to a more appropriate tool, namely cellular automata. Cellular automata and in particular lattice gas models are good candidates for studying HIS, which is one type of self organization systems(SOSs). A well known feature of CA computations is their ability to produce surprisingly complex behaviour from very simple rules. Nonlinear or time-delays is not intrinsically difficult to treat since they are either a consequence of the dynamics rather than a cause or are otherwise trivial to include in the context of the CA local rules. Another great advantage of CA is that its computational structure is inherently parallel and it can therefore be run on a parallel computer very efficiently.

\section{CONCLUSIONS}

The immune system can be seen as a parallel, information processing system that learns through examples and constantly adapts itself to new situations and possesses a distributive memory for patterns. It has many intelligent features from the point of view of AI. Many models and simulations had been established to explore its nature and its intelligent behavior. To the researchers in the field of AI, human immune system is an inspiring resource for designing new intelligent way for solving engineering problem. While to immunologists, by computer modeling and simulating, they can find new ways of improving medical approaches for people. So modeling and simulating the HIS will benefit more to both engineering and medical. The combination of them has been a trend both in the field of AI and medical, but there is still a lot of work to do.

\section{ACKNOWLEDGEMENTS}

This paper is supported by National Nature Science Foundation of China,No.60305007, and Nature Science Foundation of Heilongjiang Province, China, No.F0210 and Basic Theory Research Project Foundation of Harbin Engineering University,No.HEUF04076.

\section{REFERENCES}

[1] Bernaschi M.,Castiglione. F. Selection of escape mutants from immune recognition during HIV. Immunology and Cell Biology. Special issue devoted to Immuno-informatics. 80:307-312, 2002

[2] Bernaschi M., Castiglione F. and Succi.S. A parallel simulator of the immune response. 1401:163172,1998 
[3] Bezzi,M., Celada,F. Ruffo S. and Seiden. P.E. The transition between immune and disease states in a cellular automaton model for clonal immune response.Physica A. 245:145-163, 1997

[4] Castiglione F., Bernaschi M. and Succi S. Simulating the immune response on a distributed parallel computer.Int. J. Mod. Phys. C8:527-545, 1997

[5] Celada F.and Seiden P. A. Cmputer model of cellular interaction in the immune system. Immunology Today. 13:56-62, 1992

[6] Celada F., Seiden P.E. Affinity maturation and hypermutation in a stimulation of the humoral response Eur.J.Immunol,26(6):1350-1358, 1996

[7] Celada F., Seiden.P.E. Modeling immune cognition. Proceedings of the 1998 IEEE International Conference on Systems,Man and Cybernetics,San Diego,CA, 3787-3792,11-14 Oct.1998,

[8] De Boer,R. J., Segel L. A. and Perelson A. S. Pattern-formation in one dimensional and 2-dimensional shape space models of the immune-system. J. Theor. Biol., 155:295- 333, 1992

[9] Farmer J. D., Packard N., and Perelson A. The immune system,adaptation and machine learning. Physica D 22:187-204, 1986

[10] Forrest S., Smith R., Javornik E B and Perelson. A. S. Using genetic algorithms to explore pattern recognition in the immune system. Evolutionary Compuatation,1(30):191-211,1993

[11] Fukada T., Mori K. and Tsukiyama M. Immunity based management system for a semiconductor production line. Proc. of The 1998 International Conference on Evolutionary Computation, 1998.

[12] Hanegraaff W. Simulating the immune system. 2001 http://www.lymenet.de/literature/hanegraaff.pdf

[13] Jacob C., Litorcol J. and Lee L. Immunity Through Swarms: Agent-Based Simulations of the Human Immune System .Giuseppe N., Vincenzo C., Peter J. Bentley et al. I Artificial Immune Systems, Third International Conference, ICARIS 2004.Berlin, Springer-Heiderberg, 2004,400-412

[14] Jerne. N. Towards a network theory of the immune system. Ann. Immunol,,125C:373- 389, 1974.

[15] Joachim G. Models of immune systems: The use of differential equations.http://www.lymenet.de/literatur/immundif.htm.

[16] Kaufman M., Urbain J., and Thomas R. Towards a logical analysis of the immune response. J. Theor. Biol. 114:527, 1985.

[17] Kleinstein S. H., Seiden P. E. Simulating the immune system. Computing in Science and Engineering, 4:69-77,2000

[18] Meier-Schellersheim M., Mack.G. Simmune: a tool for simulating and analyzing immune system behavior, 1999,arXiv:cs.MA/9903017

[19] Pnadey R.,Tauffer D. Metastability with probabilistic cellular automata in an HIV infection. J. Stat. Phys. $61: 235,1990$

[20] Perelson, A. S., 1989, Immunology Review. 110, 5-36.

[21] Perelson A.S.. Hightower R. Forrest. S. Evolution and somatic learning in v-region genes. Res.Immunol., 147:202-208,1996

[22] Peter Zvirinksy A. Bottom-up Approach to Immune System Modeling. Intelligent and Adaptive system in Medicine. Prague, 20003

[23] Seiden P., Celada F. A model for simulating cognate recognition and response in the immune system. J. Theor. Biol. 158:329-357, 1992

[24] Smith D. J., Forrest S. Hightower,R. R. and Perelson A. S.. Deriving shape space parameters from immunological data. J. Theor. Biol., 189:141- 150,1997

[25] Somayaji, A. Hofmeyr,S. and Forrest.S. Principles of a computer immune system. New Security Paradigms Workshop, 1997

[26] Stauffer D., Sahimi. M. High-dimensional and very large cellularautomata for immunological shape space. Int. J. Mod. Phys. C-Phys. Comput 4:401- 408, 1993

[27] Stauffer D., Sahimi. M. High-dimensional simulation of simple immunological models. J. Theor. Biol., 166:289-297, 1994.

[28] Watanabe Y. Ishiguro A. Shirai Y. and Uchikawa Y..Emergent construction of behavior arbitration mechanisms based on the immune system. Proc. of The 1998 International Conference on Evolutionary Computation.481- 486, 1998.

[29] Zorzenon dos Santos R.M., Coutinho S..Dynamics of hiv infection:A cellular automata approach. Physical Review Letters, $87(16), 2001$ 